\title{
Prospects of searches for long-lived charged particles with MoEDAL
}

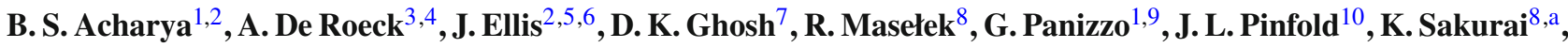 \\ A. Shaa ${ }^{8}$, A. Wall ${ }^{11}$ \\ 1 The Abdus Salam International Centre for Theoretical Physics, Strada Costiera 11, Trieste, Italy \\ 2 Theoretical Particle Physics and Cosmology Group, Department of Physics, King's College London, Strand, London WC2R 2LS, UK \\ ${ }^{3}$ Experimental Physics Department, CERN, 1211 Geneva 23, Switzerland \\ ${ }^{4}$ Antwerp University, 2610 Wilrijk, Belgium \\ 5 Theoretical Physics Department, CERN, 1211 Geneva 23, Switzerland \\ ${ }^{6}$ National Institute of Chemical Physics and Biophysics, Rävala 10, 10143 Tallinn, Estonia \\ ${ }^{7}$ School of Physical Sciences, Indian Association for the Cultivation of Science, 2A \& 2B Raja S.C. Mullick Road, Kolkata 700 032, India \\ ${ }^{8}$ Institute of Theoretical Physics, Faculty of Physics, University of Warsaw, ul. Pasteura 5, 02-093 Warsaw, Poland \\ ${ }^{9}$ INFN Gruppo Collegato di Udine, Sezione di Trieste, Udine, Italy \\ ${ }^{10}$ Physics Department, University of Alberta, Edmonton, AB T6G 2E4, Canada \\ ${ }^{11}$ Department of Physics, University of Alabama, Tuscaloosa, AL, USA
}

Received: 30 April 2020 / Accepted: 27 May 2020 / Published online: 25 June 2020

(C) The Author(s) 2020

\begin{abstract}
We study the prospects of searches for exotic long-lived particles with the MoEDAL detector at the LHC, assuming the integrated luminosity of $30 \mathrm{fb}^{-1}$ that is expected at the end of Run 3. MoEDAL incorporates nuclear track detectors deployed a few metres away from the interaction point, which are sensitive to any highly-ionizing particles. Hence MoEDAL is able to detect singly- or doublycharged particles with low velocities $\beta<0.15$ or $<0.3$, respectively, and lifetimes larger than $\mathcal{O}(1) \mathrm{m} / c$. We examine the MoEDAL sensitivity to various singly-charged supersymmetric particles with long lifetimes and to several types of doubly-charged long-lived particles with different spins and SU(2) charges. We compare the prospective MoEDAL mass reaches to current limits from ATLAS and CMS, which involve auxiliary analysis assumptions. MoEDAL searches for doubly-charged fermions are particularly competitive.
\end{abstract}

\section{Introduction}

One of the key priorities of the LHC experimental programme is to search for new particles beyond the Standard Model, or at least set robust constraints on their possible existence. Priorities during Runs 1 and 2 of the LHC included scans for bumps in invariant-mass spectra, searches for excesses of missing transverse energy in events with various topologies, and precision tests of Standard Model (SM) predictions. With the striking exception of the discovery of the Higgs boson, none of these searches has yet borne any fruit.

In parallel with these mainstream searches for new particles, there has been growing interest in less-orthodox searches. These have included searches for long-lived particles (LLPs), which we review in Sect. 2. These long-lived particles may arise in various scenarios beyond the SM that satisfy any of the following conditions: (i) small couplings, (ii) nearly degenerate masses or (iii) heavy intermediate (virtual) particles mediating decays [1-3]. We expect such searches to receive increased attention during LHC Run 3.

During Runs 1 and 2 of the LHC the search for magneticallycharged particles was the main objective of the MoEDAL experiment, which has published limits from searches for magnetic monopoles [4-7] and dyons [8]. The ATLAS Collaboration has also published limits on magnetic monopole production $[9,10]$.

As we discuss in Sect. 3 one of the key features of the MoEDAL detector [11] is its array of panels of nuclear track detectors (NTDs), which can register anomalously heavy ionization as would be produced by singly-charged particles with velocities $\beta \equiv v / c<0.15$, or by doubly-charged particles with $\beta<0.3$. The MoEDAL NTD panels are located at distances $\sim 2 \mathrm{~m}$ from the interaction point, and are therefore sensitive to long-lived heavily-ionizing particles with

a e-mails: kazuki.sakurai.1225@gmail.com; kazuki.sakurai@ fuw.edu.pl (corresponding author) 
lifetimes $\tau$ longer than $\mathcal{O}(1) \mathrm{m} / c[12,13]^{1}$. We discuss in Sect. 4 the analysis framework that we use to study longlived charged particles, which we apply to a couple of wellmotivated theoretic models in Sects. 5 and 6.

One such model is supersymmetry, which has been the object of many other searches at the LHC $[14,15]$. The minimal supersymmetric extension of the SM (the MSSM) does not contain any doubly-charged particles, but many of its singly-charged particles are candidates to be long-lived, and several mechanisms suggest this possibility [16-19]. The lightest supersymmetric particle (LSP) would be stable if Rparity is conserved, but could be unstable with a long lifetime if R-parity is weakly broken [20,21]. Even if R-parity is conserved, the next-to-lightest supersymmetric particle (NLSP) could be long-lived if it is very nearly degenerate with the LSP, as in coannihilation scenarios, or if the supersymmetric particle(s) mediating its decay is (are) very heavy, as in scenarios with split supersymmetry [22,23]. Another possibility is that the LSP has only very weak interactions, e.g., if it is the gravitino or some other particle with gravitational-strength interactions, or if it is located in a well-sequestered hidden sector. In either case, the lightest supersymmetric particle in the visible sector could be long-lived [24-26].

Prospects for discovering long-lived sparticles with MoEDAL was first discussed in $[12,13]$. These studies considered a double long-lived (LL) cascade chain: $p p \rightarrow \tilde{g} \tilde{g}$, $\tilde{g} \rightarrow j j\left[\tilde{\chi}_{1}^{0}\right]_{\mathrm{LL}},\left[\tilde{\chi}_{1}^{0}\right]_{\mathrm{LL}} \rightarrow \tau[\tilde{\tau}]_{\mathrm{LL}}$, assuming that the $\tilde{\chi}_{1}^{0}$ and $\tilde{\tau}$ are both long-lived. In this paper we extend this discussion to consider direct pair production of general meta-stable sparticles, including charged R-hadrons containing a gluino, squark or stop, as well as Winos, Higgsinos and sleptons, without making any specific assumptions about the models in which they appear [27].

A second scenario that may lead to a long-lived heavy fermion is a Type-III seesaw model [28-30], in which the SM is augmented with at least two $S U(2)_{L}$-triplet fermion fields ( $\Sigma)$ with $Y=0$. The observed neutrino mass is given by $m_{v} \approx Y_{v}^{2} v^{2} / m_{\Sigma}$, where $Y_{v}$ is the Dirac Yukawa coupling, $v$ is the SM vev and $M_{\Sigma}$ is the mass of the heavy triplet fermion. In this model, radiative corrections generate a mass splitting $m_{\Sigma^{ \pm}}-m_{\Sigma^{0}}>m_{\pi}^{ \pm}[31,32]$ so that the decay $\Sigma^{ \pm} \rightarrow \Sigma^{0}+\pi^{ \pm}$ is kinematically allowed, with a lifetime $\mathcal{O}\left(10^{-8}\right)$ s that leads to very soft charged pion but no long-lived charged-particle track. However, if there are additional contributions to the $\Sigma^{ \pm, 0}$ masses that reduce the mass difference so that the twobody decay is not allowed, the dominant decay of the $\Sigma^{ \pm}$ would be three-body with a lifetime $\mathcal{O}\left(10^{-6}\right)$ s. The latter

\footnotetext{
${ }^{1}$ Another possibility is provided by quirks [59], particles with a hidden non-Abelian charge whose confinement scale, $\Lambda$, is much lower than their vector-like mass, $m_{Q}$. After being pair-produced, quirk pairs would be restrained by a flux tube and move slowly until they finally annihilate. Electrically-charged quirks could be detected by MoEDAL, but we do not discuss them in this paper.
}

case would lead to a detectable long-lived charged particle signature similar to that of the Wino, as we discuss later in the paper.

A long-lived doubly-charged scalar particle may appear in a number of models. One example is a Type-II seesaw model of neutrino masses, in which the SM is supplemented by a complex $S U(2)_{L}$ triplet of scalar fields with hypercharge $Y=2[33-41]$, and other scenarios for doubly-charged scalars are mentioned later. Another possibility for a doublycharged particle is a spin- $1 / 2$ particle, such as a doublycharged Higgsino as appears in supersymmetric L-R models [42-46]. One can also simply add doubly-charged scalars and fermions in various $S U(2)_{L}$ representations to the particle content of the SM, then write down the SM gauge-invariant interaction terms involving these new fields and study their phenomenology $[47,48]$. We consider here doubly-charged particles that may be scalars or fermions and either singlets or triplets of the SM $S U(2)_{L}$ gauge group, without making any specific assumptions about the models in which they appear. ${ }^{2}$

Both ATLAS [53,54] and CMS [55] have set limits on certain species of long-lived supersymmetric particles, ATLAS has also considered multi-charged particles [56], and CMS [55] has also considered one example of a doubly-charged fermion as well as multi-charged particles [57], as we review in Sect. 2. However, the ATLAS and CMS searches both employed auxiliary signatures, in particular either $E_{\mathrm{T}}^{\text {miss }}$ or muon triggers [1-3]. Not all models containing long-lived charged particles would yield a large $E_{\mathrm{T}}^{\text {miss }}$ signature, and therefore the data sets used are a combination of both these complementary triggers, so as to reduce substantially the model dependence of the search. The MoEDAL analysis would not invoke any such a signatures, and hence is fully model-independent. In Table 1 we compare the prospective sensitivities of MoEDAL for a wide selection of supersymmetric candidates with the results of ATLAS and CMS, and Table 2 shows a similar comparison for a wide selection of doubly-charged candidates.

We note in passing that the parameter spaces of both the Type-II and-III seesaw models, as well as L-R symmetric models, are constrained by various theoretical considerations and low-energy experimental data [39-41,49,50,58]. Since our goal in this paper is to estimate the MoEDAL sensitivities on these exotic particles in a model-independent way, we refrain from discussing further these indirect modeldependent limits.

The outline of our paper is as follows. In Sect. 2 we review previous searches for long-lived charged particles at the LHC by ATLAS and CMS. Then, in Sect. 3 we review relevant aspects of the MoEDAL detector, and in Sect. 4 we describe the analysis framework we use. The prospective sensitivi-

\footnotetext{
2 Detailed discussions on various theoretical models of long-lived stable particles and their signatures can be found in [1-3,32,49-52].
} 
ties of MoEDAL for long-lived supersymmetric particles are obtained in Sect. 5 and those for doubly-charged particles in Sect. 6. Finally, Sect. 7 summarises our conclusions.

\section{Previous LHC searches}

There have been previous searches for long-lived particles (LLPs) at the LHC by the ATLAS $[53,54,60,61]$ and CMS $[55,57,62,63]$ Collaborations. These searches are based on observables related to ionization loss, $d E / d x$, displaced tracks, displaced vertices, delayed decays and timing information on slowly-moving massive particles. Most of these unusual events are associated with conventional final states involving photons, charged leptons, jets and missing transverse energy, $E_{T}^{\text {miss }}$. In some cases those final states occur as the decay products of LLPs, whilst in other cases they are produced in association with the LLPs. In all these searches, the $p_{T}$ of charged leptons, photons or jets and $E_{T}^{\text {miss }}$ were used to trigger the event selection.

The ATLAS and CMS collaborations classified their LLP searches into two broad classes: (i) heavy stable charged particles (HSCPs) (that may decay outside the detector) [53$55,57,60]$ and (ii) decaying LLPs [61-63]. In the first case, the HSCP gives rise to signatures that are very different from traditional prompt signatures. Singly-charged $(|Q|=e)$ HSCPs typically travel with speeds $\beta=v / c<1$, giving rise to an ionization loss $(d E / d x)$ that is different from that of minimum-ionising SM particles. Due to their slower speeds, these particles would also take an anomalously long time-of-flight (ToF) to reach the muon chamber. Using the information on ionization loss and ToF, the ATLAS and CMS collaborations set lower limits on the masses of such HSCPs, mainly in the context of supersymmetry and its variants. The ATLAS collaboration used a dataset of $36.1 \mathrm{fb}^{-1}$ collected during the $13 \mathrm{TeV}$ runs to look for such exotic particles. No significant signal events were observed over the expected background, and 95\% CL upper limits were set on the production cross-sections of long-lived R-hadrons, as well as pairs of staus and charginos. These upper limits can be translated into lower limits on the masses of a long-lived gluino, sbottom, stop, stau and chargino of 2000, 1250, 1340 and $1090 \mathrm{GeV}$, respectively [54]. ${ }^{3}$

The ATLAS Collaboration also performed a dedicated search for the anomalous ionisation signal arising from the pair production of $S U(3)_{C}$ and $S U(2)_{L}$ singlet multicharged ( $Q \mid=Z e, 2 \leq Z \leq 7)$ HSCPs with spin-1/2

\footnotetext{
${ }^{3}$ A shorter-lived coloured LSP could decay inside a detector after hadronization to form an R-hadron, e.g., a gluino hadron may decay into pair of SM quark jets and the lightest neutralino, $\chi_{1}^{0}$. Using the same data set, the ATLAS Collaboration set a lower mass limit of 1290-2060 $\mathrm{GeV}$ on the gluino, assuming pair production followed by such a decay with $m_{\chi_{1}^{0}}=100 \mathrm{GeV}$ [53].
}

in the mass range from 50 to $1400 \mathrm{GeV}$ using an integrated luminosity of $36.1 \mathrm{fb}^{-1}$ [56]. Non-observation of any significant deviation from the standard model background translates into 95\% CL upper limits on the Drell-Yan pair production cross-section as a function of the charge of a lepton-like HSCP for several values of $Z$ between 2 and 7. This upper limit on the pair production cross-section can be recast into 95\% CL lower limits on the masses of lepton-like HSCPs for charges $|Q|=Z e: 2 \leq Z \leq 7$, ranging between 980 and $1220 \mathrm{GeV}$ for $Z=2$ and $Z=7$, respectively. The CMS Collaboration studied the pair production of $S U(3)_{C}$ and $S U(2)_{L}$ singlet singly-charged $(Z=1)$, multiply-charged $(Z>1)$ and fractionally-charged $(Z<1)$ spin-1/2 HSCPs produced via the Drell-Yan process during Run 1 of the LHC at $\sqrt{s}=7$ and $8 \mathrm{TeV}$, with integrated luminosities of $5 \mathrm{fb}^{-1}$ and $18.8 \mathrm{fb}^{-1}$ respectively. The $95 \% \mathrm{CL}$ lower limit on the masses are $480(Z=2 / 3), 574(Z=1), 685(Z=2), 796$ $(Z=5), 781(Z=6), 757(Z=7)$ and $715(Z=8) \mathrm{GeV}$, respectively [57].

The CMS Collaboration also put 95\% CL lower mass bounds on the gluino, stop and stau of 1610, 1040 and $490 \mathrm{GeV}$, respectively, from the production of HSCPs at the $13 \mathrm{TeV}$ LHC run using a data sample from an integrated luminosity of $2.5 \mathrm{fb}^{-1}$. The corresponding mass limits on $|Q|=1 e$ (2e) lepton-like fermions of $550(680) \mathrm{GeV}$ were obtained by the CMS collaboration [55]. ${ }^{4}$

\section{The MoEDAL detector}

The MoEDAL detector [11] is located at Point 8 of the LHC, around the interaction point in the VErtex LOcator (VELO) cavern of the LHCb detector. MoEDAL is largely a passive LHC detector, and the subdetector system of principal relevance for this analysis is comprised of a large array $\left(120 \mathrm{~m}^{2}\right)$ of nuclear track detector (NTD) stacks composed of CR39 and Makrofol plastic surrounding the interaction region. MoEDAL also has paramagnetic trapping volumes (MMTs) that can capture highly-ionizing electricallyand magnetically-charged particles. The possible decays of trapped long-lived electrically-charged particles can be monitored at a remote facility, and magnetically-charged particles are monitored at the ETH Zurich SQUID Magnetometer Facility. MoEDAL also incorporates an array of TimePix

\footnotetext{
$\overline{4}$ A dedicated search by the CMS Collaboration for an anomalous signal arising from disappearing charged tracks using the data collected in Run 2 during the $13 \mathrm{TeV}$ run of the LHC, corresponding to an integrated luminosity of $140 \mathrm{fb}^{-1}$, set a 95\% CL lower limit $m_{\chi_{1}^{ \pm}}=880(700) \mathrm{GeV}$ for a purely wino LSP in the AMSB model $[24,25]$ for $\tau_{\chi_{1}^{ \pm}}=3$ (33) ns, respectively [63]. The CMS Collaboration used a similar signature and $35.9 \mathrm{fb}^{-1}$ of data from the $13 \mathrm{TeV}$ LHC run [64] to constrain the $S U(2)_{L}$-triplet charged fermion mass $m_{\Sigma^{ \pm}}$in the Type-III Seesaw model [28] to be $>840 \mathrm{GeV}$.
} 
pixel devices that serves as a real-time system for monitoring highly-ionizing backgrounds in the cavern. We do not consider the MMTs and TimePix devices in this analysis, but focus on the capabilities of the NTD stacks.

The NTD elements of the MoEDAL detector are passive, not needing a trigger, electronic readout, high-voltage or gas system. They are calibrated directly for highly-ionizing particles by using heavy-ion beams. Thus, they complement the main LHC detectors, ATLAS and CMS, which are not optimized for detecting heavily-ionizing particles. The NTD array has a low threshold and can detect particles with an ionization level $Z / \beta \sim 7$, where $Z$ is the electric charge and $\beta$ is the velocity of the particle. The charge resolution of the plastic NTDs is better than $0.05 e$, where $e$ is the electric charge. The TDR NTD array is supplemented by a very high charge catcher (VHCC) subdetector with threshold $Z / \beta \sim 50$ applied directly to the outside of the VELO detector housing so as to increase the geometrical acceptance for magnetic monopoles, which does not play a role in our analysis.

After the NTD sheets are etched they must be scanned using optical microscopes, since the feature (etch-pit) sizes lie in the range 20 to $50 \mu \mathrm{m}$. A computer-controlled optical scanning microscope system will be deployed for MoEDAL data taking during Run 3 . The system will be controlled by dedicated artificial intelligence (AI) software that is designed to recognize signal etch-pits in the presence of beam-induced backgrounds.

The multi-sheet structure of the NTD stack enables the measurements of individual pits to be combined to define a precise trajectory and effective $Z / \beta$ values that measure the change in ionization energy loss as the particle loses energy during its passage through the NTD detector stack, demonstrating that the track comes from the interaction region and has $d E / d x$ values consistent with a heavily-ionizing electrically-charged particle.

These characteristics of the NTD system ensure that such a particle can be detected with high efficiency and confidence within the geometric acceptance of the detector.

\section{Analysis framework}

In this study we consider the pair production of singly- and doubly-charged particles at the LHC, $p p \rightarrow Y \bar{Y}+X$, where $\bar{Y}$ represents the antiparticle of $Y$ (which is same as $Y$ if it is self-conjugate), and $X$ represents soft particles originating from the beam remnants and initial- and final-state QCD radiation. The particle $Y$ may be a supersymmetric particle, specifically an R-hadron containing the strongly-interacting $\tilde{g}, \tilde{q}, \tilde{t}$, a chargino $\tilde{\chi}^{ \pm}$or a charged slepton $\tilde{\ell}^{ \pm}$, or a doublycharged particle with spin- $0, S^{++}$, or spin- $1 / 2, f^{++}$.

As already discussed, the MoEDAL detector is insensitive to electrically-neutral particles and a coloured supersymmet- ric particle must be hadronized into a charged $\mathrm{R}$-hadron to be detected. Since this probability $\kappa$ (the charged vs neutral R-hadron fraction) is not well understood [65], we vary this parameter over the range $\kappa \in[0.5,0.7]$. The distances between the interaction point and the MoEDALS's NTD panels are on average $\sim 2 \mathrm{~m}$, so MoEDAL is sensitive to particles $Y$ with lifetimes $\tau$ longer than $\mathcal{O}(1) \mathrm{m} / c$, otherwise the detection probability is exponentially suppressed. In this study we treat $\tau$ as a free parameter and in the next Section we identify the region of the mass vs lifetime plane for each choice of $Y$ that can be probed by MoEDAL with Run 3 data.

As also discussed in the previous section, the MoEDAL detector is essentially free of SM backgrounds, and we are therefore interested in the regions of parameter space where the numbers of expected signal events detected by MoEDAL are $N_{\text {sig }} \geq 1$ and 2, which we define as the thresholds for "evidence" and "discovery". The numbers of expected signal events are estimated by Monte Carlo simulation using the following formula:

$N_{\text {sig }}(m, \tau)=\sigma(m) \cdot L \cdot\left\langle\sum_{i} \Theta\left(\beta_{\mathrm{th}}-\beta_{i}\right) P\left(\vec{\beta}_{i}, \tau\right)\right\rangle_{\mathrm{MC}}$,

where $\sigma(m)$ is the production cross-section as a function of the target particle's mass, $m, L$ is the integrated luminosity, $\vec{\beta}$ is the particle's three-velocity, and $\langle\cdots\rangle_{\text {MC }}$ represents the Monte Carlo average. The summation over $i$ includes the two target particles $Y$ and $\bar{Y}$ in the event. The Heaviside step function $(\Theta(x)=1$ for $x>0$ and 0 otherwise) models the fact that the NTDs are capable to detect a particle with ionization level higher than some threshold, $Z / \beta>1 / \beta_{\text {th }}$.

The threshold velocity $\beta_{\text {th }}$ for detection depends in principle on the incident angle between the direction of the particle and the NTD panel. This effect was significant in Run 2 of the LHC, because the NTD panels were not ideally oriented (see [12] for a study of this effect). However, in Run 3 all the NTD panels will be facing the interaction point and the incident angles are always close to optimal. We therefore use a constant threshold velocity $\beta_{\text {th }}$ in this study for Run 3 . We take $\beta_{\text {th }}=0.15(0.3)$ for singly- (doubly-)charged particles.

We denote the probability for the target particle with threevelocity $\vec{\beta}$ and lifetime $\tau$ to survive and hit an NTD panel by $P\left(\vec{\beta}_{i}, \tau\right)$, which can be expressed as

$P(\vec{\beta}, \tau)=\epsilon(\vec{\beta}) \cdot \exp \left(-\frac{l(\vec{\beta})}{\gamma \beta c \tau}\right)$.

In this expression $\epsilon(\vec{\beta})=1$ if there is an NTD panel in the direction of $\vec{\beta}$ and 0 otherwise. The exponential factor represents the probability that the target particle does not decay before reaching an NTD panel, where $l(\vec{\beta})$ is the distance between the interaction point and the NTD panel in the direction of $\vec{\beta}, \tau$ is the lifetime and $\gamma=\left(1-\beta^{2}\right)^{-1 / 2}$. 
In order to evaluate the Monte Carlo average in Eq. (1) we used Madgraph5_aMC v-2.6.7 [66], and implemented several types of doubly-charged particles using FeynRules 2 [67].

\section{Singly-charged sparticles}

\subsection{Motivations for candidates}

Candidates for the long-lived sparticle include the gluino, squarks, Winos and Higgsinos, and sleptons.

The gluino is not detectable directly, since it hadronizes very soon after production to form colour-neutral hadrons. The colour of the gluino may be neutralized by a gluon, forming a neutral hadron, or by a colour-octet quark pair. We focus on the latter possibility, assuming that the gluinogluon state is heavier. We further assume that gluino/firstgeneration quark states $\tilde{g} u \bar{u}, \tilde{g} d \bar{d}, \tilde{g} u \bar{d}$ and $\tilde{g} d \bar{u}$ are the lightest. Depending on the details of superhadronic spectroscopy, the lightest of all the gluino/quark states may be neutral or charged. In the former case MoEDAL would not see a signal: we consider here the latter case.

We consider separately the possibilities that the long-lived sparticle is the lightest squark, which may be a partner of one of the 5 lightest quarks, or a stop. We assume that in the former case the differences between these squark masses would be relatively small, with the stops much heavier, whereas in the light stop case the other squarks would be much heavier. In both cases, we assume that the other squark species decay into the lightest one on a time scale $\ll \mathcal{O}(1) \mathrm{m} / c$. The resultant long-lived squark would also appear as a bound state, of which the lightest is expected to be that with a firstgeneration antiquark, e.g., $\tilde{u} \bar{u}, \tilde{d} \bar{d}, \tilde{u} \bar{d}$ or $\tilde{d} \bar{u}$ if the lightest squark is associated with the first generation. In the former two cases the lightest squark hadron would be neutral, and MoEDAL would not see a signal: we assume here one of the latter cases of a charged long-lived particle.

Another possibility is that the long-lived sparticle is some mixture of Wino, $\widetilde{W}$, and Higgsino, $\tilde{h}$, and we consider the cases where one or the other component dominates production. Decays into the lightest $\widetilde{W} / \tilde{h}$ combination would again occur on a time scale $\ll \mathcal{O}(1) \mathrm{m} / c$, and this combination could be either neutral or charged: we consider the latter case here.

We consider finally the possibility that the long-lived sparticle is a charged slepton, $\tilde{\ell}$, and our results apply to any flavour of slepton, assuming that the other slepton flavours are much heavier. The lightest slepton might be the supersymmetric partner of either a left- or right-handed lepton, and we consider both possibilities here, making the conservative assumption that the heavier one is heavy enough to be effectively decoupled. In what follows we refer to the light- est slepton as the $\tilde{\tau}_{L / R}$, since the tau slepton is the lightest in many supersymmetric models ${ }^{5}$. However, it should be noted that the numerical results for $\tau_{L / R}$ are also applicable to the other sleptons, namely $\tilde{e}_{L / R}$ and $\tilde{\mu}_{L / R}$, as long as they are the lightest and other states are decoupled. We recall that the detection with MoEDAL of long-lived sleptons via the cascade decays of long-lived gluinos was studied in [12,13], whereas in this Section we consider the direct pair production of long-lived sleptons.

\subsection{Run 3 projections for supersymmetric particles}

We begin the discussion of MoEDAL's projected sensitivity at Run 3 for singly-charged long-lived supersymmetric particles, showing in the upper panel of Fig. 1 the $13 \mathrm{TeV}$ cross-sections for pair production of various supersymmetric particle species. The cross-sections are all taken from the LHC SUSY Cross Section Working Group [71], except for $\tilde{\tau}_{L}$, for which we used Resummino 2 . 0.1 [73]. The cross-sections for coloured supersymmetric particles $\left(\tilde{g}, \tilde{q}\right.$ and $\left.\tilde{t}_{1}\right)$ are computed including approximate next-tonext-to-leading order $\left(\mathrm{NNLO}_{\text {Approx }}\right)$ supersymmetric QCD corrections and the resummation of soft gluon emission at next-to-next-to-leading-logarithmic (NNLL) accuracy [72], whereas those for the weakly-interacting supersymmetric particles $\left(\tilde{W}, \tilde{h}\right.$ and $\left.\tilde{\tau}_{L / R}\right)$ are calculated to NLO+NLL accuracy.

The curve for $\tilde{q}$ in the upper panel of Fig. 1 is the sum of the cross-sections for both the left- and right-handed versions of all 5 light-flavoured squarks, calculated assuming $m_{\tilde{g}}=3 \mathrm{TeV}$. In the cases of electroweakly-interacting sparticles, the cross-sections are summed over all triplet (doublet) components for $\widetilde{W}\left(\tilde{h}\right.$ and $\tilde{\tau}_{L}$ ). Namely, we included $\widetilde{W}^{ \pm} \widetilde{W}^{0}$ and $\widetilde{W}^{+} \widetilde{W}^{-}$production for Winos, $\tilde{h}^{+} \tilde{h}^{-}, \tilde{h}_{1,2}^{0} \tilde{h}^{ \pm}$and $\tilde{h}_{1}^{0} \tilde{h}_{2}^{0}$ production for Higgsino and $\tilde{\tau}_{L}^{+} \tilde{\tau}_{L}^{-}, \tilde{v}_{\tau} \tilde{\tau}_{L}^{ \pm}$and $\tilde{v}_{\tau} \tilde{v}_{\tau}$ for producing the left-handed slepton, assuming that the heavier components of the multiplets decay promptly into the lightest charged partner, which we assume to be long-lived. ${ }^{6}$

As expected, one can see in Fig. 1 that the coloured sparticles have the largest cross-sections, while sleptons $\tilde{\tau}_{L / R}$ have smaller cross-sections. For cross-sections to be above $1 \mathrm{pb}$, the masses have to be smaller than around $1 \mathrm{TeV}(\tilde{g}), 700$ $\operatorname{GeV}\left(\tilde{q}, \tilde{t}_{1}\right), 400 \mathrm{GeV}(\tilde{W}, \tilde{h})$ and $200 \mathrm{GeV}\left(\tilde{\tau}_{L / R}\right)$.

As already mentioned, the production velocity of a singlycharged particle must be $<\beta_{\text {th }}=0.15$ for it to be detectable by MoEDAL's NTDs. In the lower panel of Fig. 1 we

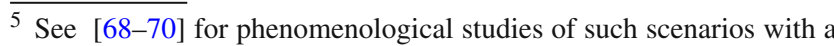
long-lived $\tilde{\tau}$.

${ }^{6}$ Charged Winos are usually heavier than the neutral one by an amount $>m_{\pi^{ \pm}}$, due to radiative corrections. However, the mass ordering can be reversed by tuning the mixing in the chargino and neutralino sectors, which is the scenario considered in this study.
} 


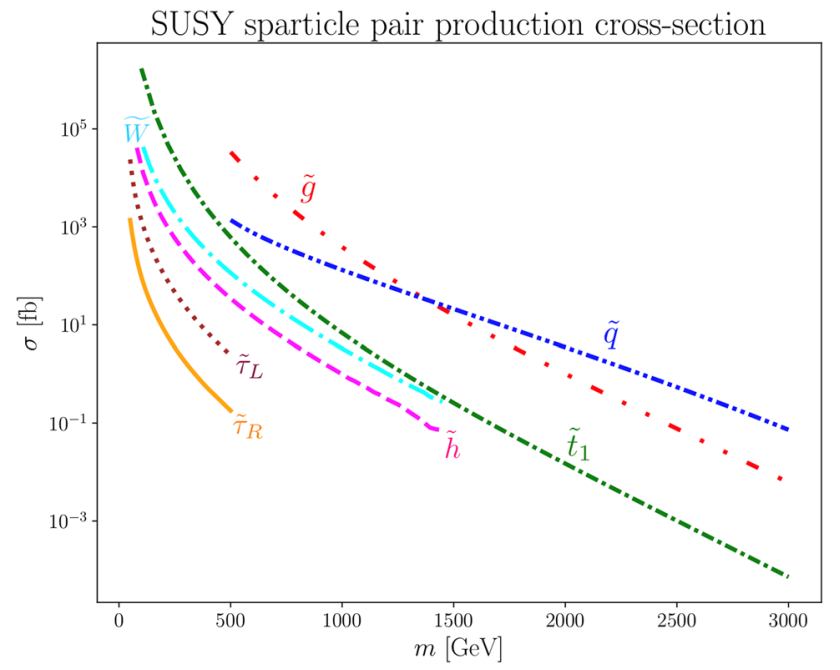

Fig. 1 Upper panel: the production cross-sections for various sparticle species. For the coloured particles $\left(\tilde{g}, \tilde{q}\right.$ and $\left.\tilde{t}_{1}\right)$, we show $\mathrm{NNLO}_{\text {Approx }}+\mathrm{NNLL}$ cross-sections [71], while the cross-sections plotted for the weakly-interacting particles $\left(\tilde{W}, \tilde{h}\right.$ and $\left.\tilde{\tau}_{L / R}\right)$ are calculated to NLO+NLL accuracy [72]. Lower panel: the velocity distributions for various sparticle species. The following representative masses were chosen: $\tilde{g}$ : $1010 \mathrm{GeV}, \tilde{q}: 920 \mathrm{GeV}, \tilde{t}_{1}: 720 \mathrm{GeV}, \widetilde{W}: 300 \mathrm{GeV}, \tilde{\tau}_{L}: 80$ $\mathrm{GeV}$

show the normalised velocity distributions for various sparticle species, as computed with MadGraph5_aMC. We took the following representative masses for this purpose: $\tilde{g}$ : $1010 \mathrm{GeV}, \tilde{q}: 920 \mathrm{GeV}, \tilde{t}_{1}: 720 \mathrm{GeV}, \widetilde{W}: 300 \mathrm{GeV}, \tilde{\tau}_{L}: 80 \mathrm{GeV}$. The velocity distribution of the right-handed slepton $\tilde{\tau}_{R}$ (not shown) is similar to that shown for the $\tilde{\tau}_{L}$.

We see that the coloured sparticles have, in general, much lower velocities than the weakly-interacting sparticles. One reason for this is that the masses used in this calculation are larger for coloured particles than for weakly-interacting ones, so their production is more central. Another effect is that the production of coloured particles may be dominated by the gluon-gluon-initiated $t$-channel process. On the other hand, the production of weakly-interacting particles is largely dominated by the Drell-Yan $s$-channel process from a quarkantiquark initial state. We also observe that the fermionic particles $\widetilde{W}$ and $\tilde{h}$, have much lower velocities than the scalar particle $\tilde{\tau}_{L}\left(\tilde{\tau}_{R}\right)$ on average. This is because the $s$-channel process is mediated by spin- 1 gauge bosons $\left(\gamma / Z / W^{ \pm}\right)$, and the pair-production rate for scalar particles vanishes in the limit of $\beta \rightarrow 0$ because of $p$-wave suppression, which is absent for the pair production of the fermionic particles $\widetilde{W}$ and $\tilde{h}$.

The grey vertical line in the lower panel of Fig. 1 marks the detection threshold velocity $\beta_{\text {th }}=0.15$, and the portions of the distributions to the left of this line can be detected. We see that the detection efficiency is highest for coloured supersymmetric particles, much lower for the slepton, and intermediate for $\widetilde{W}$ and $\tilde{h}$.
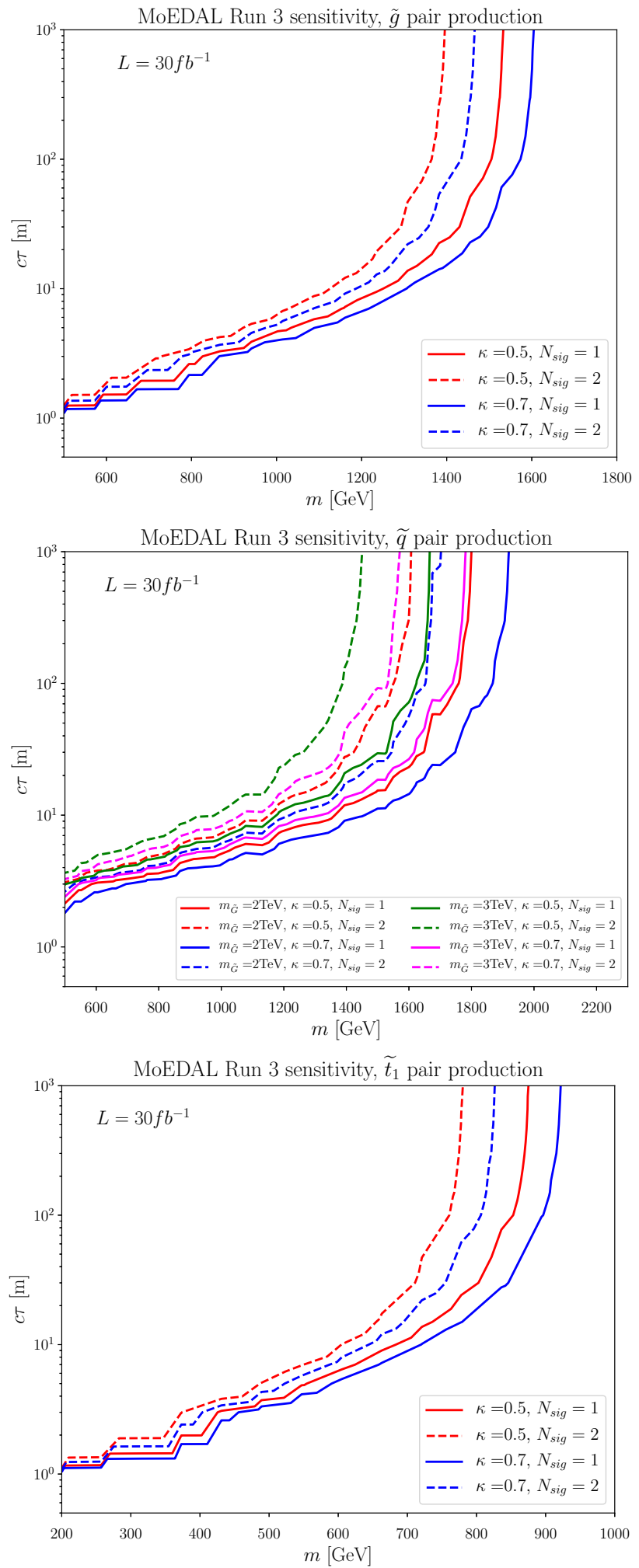

Fig. 2 The expected sensitivities of MoEDAL for various long-lived strongly-interacting sparticle species, assuming $30 \mathrm{fb}^{-1}$ of integrated luminosity. Top panel: the gluino, middle panel: squarks, bottom panel: the stop squark 
We now discuss the expected sensitivities of MoEDAL to long-lived supersymmetric particles in Run 3 of the LHC, assuming an integrated luminosity of $30 \mathrm{fb}^{-1}$. In Fig. 2 we show the contours of $N_{\text {sig }}=1$ (solid) and 2 (dashed), corresponding to the thresholds for "evidence" and "discovery", respectively, for strongly-interacting sparticles in mass versus $c \tau$ planes. The top panel shows the sensitivities for gluinos with the red and blue contours corresponding to the charged R-hadron fractions of $\kappa=0.5$ and 0.7 , respectively. Under the optimistic assumptions $\kappa=0.7$ and $c \tau \gtrsim 100 \mathrm{~m}$, MoEDAL is expected to see 1 (2) signal event(s) for $m_{\tilde{g}} \simeq 1600$ (1470) $\mathrm{GeV}$, while in the more conservative case $\kappa=0.5$, the mass reach is $\sim 1530$ (1400) GeV for $N_{\text {sig }}=1(2)$.

The middle panel of Fig. 2 shows the MoEDAL sensitivity to light-flavour squarks. Since the production cross-section depends on $m_{\tilde{g}}$, we show results for two gluino masses, $m_{\tilde{g}}=$ $2 \mathrm{TeV}$ for $\kappa=0.5$ (red) and $\kappa=0.7$ (blue) and $3 \mathrm{TeV}$ for $\kappa=0.5$ (green) and $\kappa=0.7$ (magenta). We see from the plot that the mass reach is greater for the smaller gluino mass, since the cross-section for light-flavour squark production is larger in this case. In the most optimistic case $\left(m_{\tilde{g}}=2 \mathrm{TeV}\right.$, $\kappa=0.7, c \tau \gtrsim 100 \mathrm{~m})$, MoEDAL could detect the gluino up to $\sim 1920$ (1700) $\mathrm{GeV}$ for $N_{\text {sig }}=1$ (2). On the other hand for $m_{\tilde{g}}=3 \mathrm{TeV}$ and $\kappa=0.5$, the mass reach is $\sim 1670$ (1450) $\mathrm{GeV}$ for $N_{\text {sig }}=1$ (2).

The Run 3 sensitivity for the lighter stop, $\tilde{t}_{1}$, is presented in the bottom panel of Fig. 2, where the convention for the linestyles is the same as in the top panel. We see that a long-lived $\tilde{t}_{1}$ could be probed by MoEDAL up to $m_{\tilde{t}_{1}} \sim 920\left(N_{\text {sig }}=1\right)$ and $830\left(N_{\text {sig }}=2\right) \mathrm{GeV}$ for $\kappa=0.7$, while the reach is $\sim 870\left(N_{\text {sig }}=1\right)$ and $780\left(N_{\text {sig }}=2\right) \mathrm{GeV}$ for $\kappa=0.5$.

We now turn to discuss the expected sensitivities for weakly-interacting sparticle species shown in Fig. 3. The Run 3 projections for MoEDAL searches for a long-lived Wino (blue) and Higgsino (red) are shown in the top panel. We see that MoEDAL could probe the Wino up to $m_{\widetilde{W}} \sim 670$ (570) $\mathrm{GeV}$ for $N_{\text {sig }}=1$ (2) if $c \tau \gtrsim 100 \mathrm{~m}$, whereas the mass reach for the Higgsino with $c \tau \gtrsim 100 \mathrm{~m}$ is $m_{\tilde{h}} \sim 530$ (430) $\mathrm{GeV}$ for $N_{\text {sig }}=1$ (2). The higher mass reach for the Wino is obtained because the cross-section is larger thanks to its larger SU(2) charge.

The bottom panel of Fig. 3 shows the MoEDAL Run 3 sensitivities for long-lived sleptons, $\tilde{\tau}_{R}$ (blue) and $\tilde{\tau}_{L}$ (red). As can be seen, the mass reach is very low: it is 61 (58) GeV for the meta-stable $\tilde{\tau}_{L}$ with $N_{\text {sig }}=1$ (2), and $56 \mathrm{GeV}$ for $\tilde{\tau}_{R}$ with $N_{\text {sig }}=1$ (there is no corresponding contour for $N_{\text {sig }}=2$ for $\tilde{\tau}_{R}$.). All of these reaches are below limits established at LEP [74] and already excluded. The reason for the low sensitivity is twofold. First, as discussed above the cross-sections for slepton production are very low, since production is mediated by spin- 1 gauge bosons in the $s$-channel process and the production rate is velocity suppressed. Secondly, for the
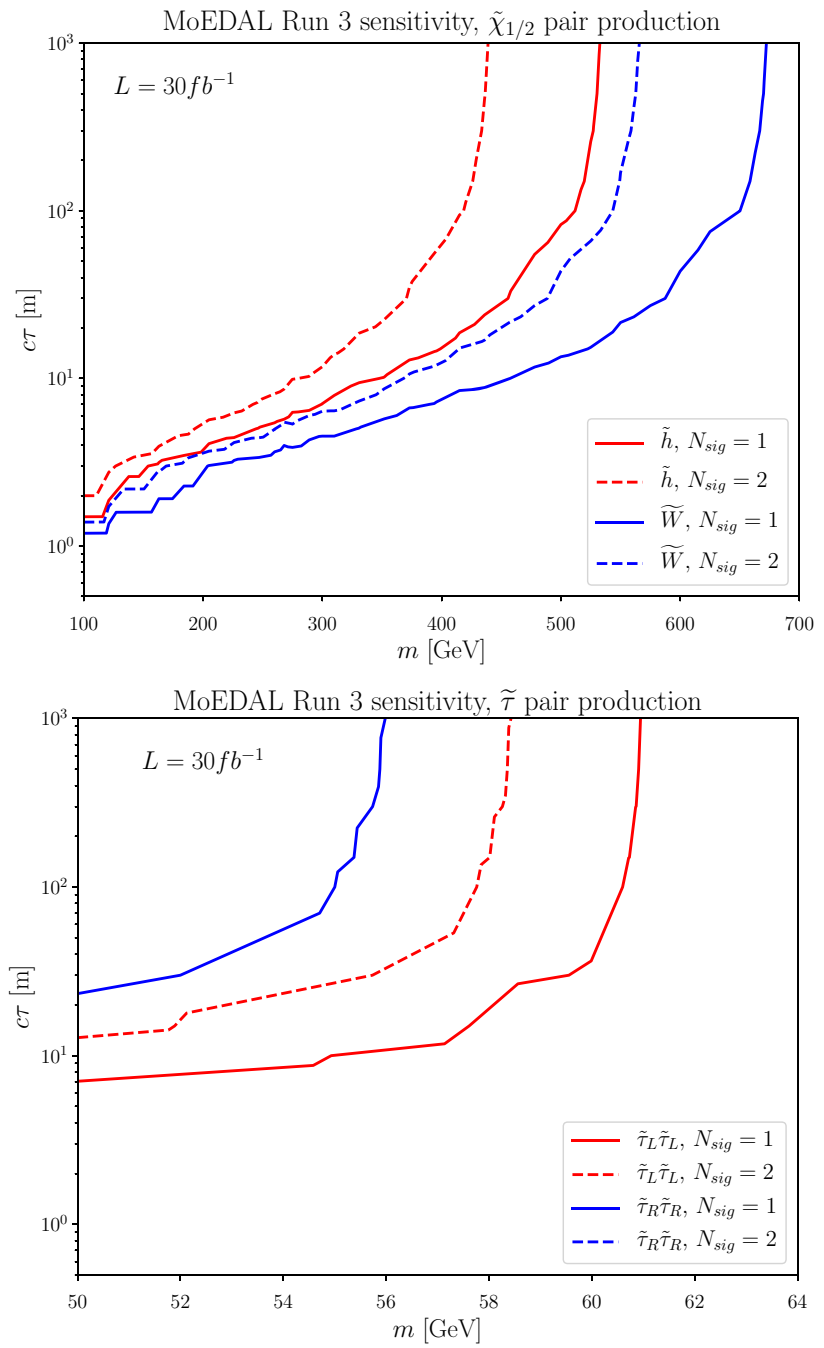

Fig. 3 The expected sensitivities of MoEDAL for various long-lived electroweakly-interacting sparticle species, assuming $30 \mathrm{fb}^{-1}$ of integrated luminosity. Upper panel: charginos, lower panel: sleptons

same reason the produced sleptons must have larger velocities than the other sparticle species examined here, as was also discussed previously. We conclude that, since MoEDAL targets highly-ionizing particles with $Z / \beta \gtrsim 7$, it is not sensitive to sleptons.

\subsection{Comparison with the existing searches}

We now compare the prospective MoEDAL sensitivities at Run 3 with the existing mass limits for metastable supersymmetric particles. Several different types of long-lived signatures have been discussed in the literature and searched for by ATLAS and CMS. For example, if gluinos have a lifetime of $1-100 \mathrm{~mm} / \mathrm{c}$ and decay into dijets, they can be searched for by reconstructing the displaced vertices originating from meta-stable gluino decay $[60,62]$. Another example is a disappearing track signature from long-lived winos with $\tau \sim 1$ - 
Table 1 Comparison between the MoEDAL mass reaches at Run 3 for $N_{\text {sig }}=1, \kappa=0.7$ and (in parentheses) the current (95\% CL) mass bounds on several long-lived supersymmetric particle candidates obtained (estimated in the cases of $\tilde{q}$ and $\tilde{h}$, in double parentheses) from the ATLAS heavy stable charged particle search with $L=36.1 \mathrm{fb}^{-1}$ [54] (second column) and CMS [55] (third column) under the modeldependent assumptions described there and in the text. All masses are in $\mathrm{GeV}$

\begin{tabular}{llll}
\hline & MoEDAL & (ATLAS) & (CMS) \\
\hline$\tilde{g}$ & 1600 & $(2000)$ & $(1500)$ \\
$\tilde{q}$ & 1920 & $((2310))$ & - \\
$\tilde{t}$ & 920 & $(1350)$ & $(1000)$ \\
$\tilde{W}$ & 670 & $(1090)$ & - \\
$\tilde{h}$ & 530 & $((1170))$ & - \\
$\tilde{\tau}$ & 61 & $(430)$ & $(230)$ \\
\hline
\end{tabular}

$10 \mathrm{~cm} / \mathrm{c}$. The long-lived signature emerges when a charged wino is produced and decays into a nearly mass-degenerate neutral partner inside one of the silicon track detectors $[61,63]$. Although the aforementioned searches are powerful for specific scenarios, they are not relevant for particles with longer lifetimes, or when the dominant decay modes differ from those assumed in the analyses. Since the MoEDAL search is independent of the long-lived particle decay mode after passing through the NTDs, it avoids the model dependence of the above constraints.

There is, however, one type of search whose constraints are less model-dependent. These are heavy stable charged particle (HSCP) searches, which rely on large ionization energy loss $d E / d x$, the MoEDAL signature considered here, and time-of-flight $(\mathrm{ToF})$ measurements, both of which are independent of the nature of decays. ${ }^{7}$ We therefore focus now on the constraints obtained from the HSCP searches.

In Table 1 we summarise the prospective MoEDAL mass reaches at Run 3 for $N_{\text {sig }}=1, \kappa=0.7$ and compare them with the current (95\% CL) mass limits obtained by the most recent ATLAS HSCP analysis with $L=36.1 \mathrm{fb}^{-1}$ [54]. The ATLAS Collaboration interpreted their results for longlived gluino, stop, Wino and stau candidates, and derived the lower bounds on their masses shown in parentheses in the third column of Table 1. At the time of writing, the most recent CMS HSCP analysis [55] is based on a smaller set of data $\left(L=2.5 \mathrm{fb}^{-1}\right)$ and its limits on the gluino, stop and stau are weaker than those of ATLAS, as seen in the last column of Table 1.

The ATLAS constraints on the $\tilde{g}, \tilde{t}$ and $\tilde{W}$ long-lived particle candidates are nominally stronger than the prospective reaches of MoEDAL with Run 3 data. However, the ATLAS selection cuts for this search may lead to gaps in the full

\footnotetext{
7 A scenario that can relax the constraint from HSCP analyses has been proposed and studied in [54].
}

phase space coverage that can be avoided by MoEDAL. As summarised in Table 1 of [54], ATLAS defined 5 signal regions (SRs), all of which incorporated $E_{\mathrm{T}}^{\text {miss }}$ triggers (varying between 70 and $110 \mathrm{GeV}$ ) or single (isolated) highmomentum muons. ${ }^{8}$ Depending on the scenario for longlived sparticles that is considered, either or both of these auxiliary signatures may be absent. For example, there would be no $E_{\mathrm{T}}^{\text {miss }}$ signature in a scenario with weak R-parity violation, so these events would need to get selected by a muon-like signature in the detector.

We note also that in [54] ATLAS did not interpret their result in scenarios with long-lived light-flavour squarks or Higgsinos. In order to estimate the possible ATLAS mass reaches for these particles, we recast the cross-section upper limits for sbottoms (Winos) derived by ATLAS [54]. Our recasting assumes that the detection efficiencies of the HSCP analysis would be similar for light-flavour squarks and bottom squarks, and for Winos and Higgsinos. Based on this assumption we derive the mass bounds 2310 (1170) $\mathrm{GeV}$ for squarks (Higgsinos) shown in double parentheses in Table 1.

Comparing the prospective sensitivities of MoEDAL for long-lived sparticles with those of ATLAS and CMS, we note the following two points. One is that the luminosity to be accumulated by MoEDAL is only about one fifth of that already accumulated by ATLAS or CMS. This is because MoEDAL is located at Point 8 of the LHC together with the $\mathrm{LHCb}$ detector, which requires a restricted instantaneous luminosity so as to suppress pile-up. ${ }^{9}$ Secondly, MoEDAL is sensitive only to particles with the ionization levels higher than $Z / \beta \gtrsim 7$, which reduces the acceptance for singlycharged HSCPs, in particular.

Finally, we comment on the case of an $S U(2)_{L}$-triplet fermion, $\left(\Sigma^{ \pm}, \Sigma^{0}\right)$, in a Type-III seesaw model, as was briefly mentioned in Sect. 1. In general, the mass degeneracy between $\Sigma^{ \pm}$and $\Sigma^{0}$ is resolved due to radiative corrections in such a way $m_{\Sigma^{ \pm}}-m_{\Sigma^{0}}>m_{\pi}^{ \pm}$, which makes the lifetime of $\Sigma^{ \pm}$too short for an HSCP signal in MoEDAL. However, if there are additional contributions to the mass splitting so that $m_{\Sigma^{ \pm}}-m_{\Sigma^{0}}<m_{\pi}^{ \pm}$, the lifetime of $\Sigma^{ \pm}$would be long enough for detection of $\Sigma^{ \pm}$at MoEDAL to be possible. For $m_{\Sigma^{0}}>m_{\Sigma^{ \pm}}+m_{\pi}^{ \pm}, \Sigma^{0}$ decays promptly to $\Sigma^{ \pm}$and the mass reach would be $\sim 670 \mathrm{GeV}$ and the same as that for the Winos studied in this section. For $m_{\Sigma^{0}}+m_{\pi}^{ \pm}>m_{\Sigma^{ \pm}}>m_{\Sigma^{0}}, \Sigma^{ \pm}$ undergoes three-body decay to $\Sigma^{0}$ with a lifetime $\mathcal{O}\left(10^{-6}\right) \mathrm{s}$. In this case, the $\Sigma^{0}$ does not contribute to the signal and the signal yield would be reduced by roughly $1 / 2$, since the dominant production mode is $p p \rightarrow \Sigma^{ \pm} \Sigma^{0}$. We expect that the

\footnotetext{
$\overline{8 \text { Similarly, the }}$ CMS analysis [55] incorporates an $E_{\mathrm{T}}^{\text {miss }}>170 \mathrm{GeV}$ requirement.

${ }^{9}$ On the positive side, this restriction results in lower beam-related background in the MoEDAL NTD detectors.
} 
mass reach in this case would be similar to that for the Higgsino studied in this section.

\section{Doubly-charged particles}

\subsection{Motivations for candidates}

Doubly-charged scalars can arise in variety of scenarios for physics beyond the standard model (BSM). As mentioned in Sect. 1, the Type-II seesaw model can provide doublycharged scalars $\left(H^{ \pm \pm}\right)$[33-38] that will be relevant for this analysis. In addition to the Type-II seesaw model, several other BSM scenarios, namely the Left-Right model [7577], the Georgi-Machacek (GM) model [78-82], the 3-31 model [83,84] and the little Higgs model [85] also predict doubly-charged scalars. The supersymmetric versions of these models can lead to doubly-charged spin-1/2 Higgsinos. The minimal left-right supersymmetric model which is based on $S U(3)_{C} \times S U(2) L \times S U(2)_{R} \times U(1)_{B-L}$ is one such model [42-46]. In addition to these particular well-studied models for multiply-charged scalars and fermions, such particles can also arise in the simplified models discussed in $[47,48]$.

In the pure Type-II seesaw model, the doubly-charged scalars $\left(H^{ \pm \pm}\right)$belonging to the $S U(2)_{L}$-triplet scalar multiplet $\Delta$ couple to leptons and the $W$ boson, and the corresponding interaction strengths are controlled by the vacuum expectation value $\left(v_{T}\right)$ of the neutral component of the $\Delta$. This vev is related to the Majorana masses of the neutrinos: $M_{v}=\sqrt{2} Y_{v} v_{T}$, where $Y_{v}$ denotes the neutrino Yukawa couplings, and the collider limits on the the mass of the doubly-charged scalars depend on the value of $v_{T}$. For small $v_{T} \leq 10^{-4} \mathrm{GeV}$ that corresponds to large $Y_{\nu}$, and assuming degenerate heavy scalar $\left(A, H, H^{ \pm}, H^{ \pm \pm}\right)$ masses, $\operatorname{Br}\left(H^{ \pm \pm} \rightarrow \ell^{ \pm} \ell^{ \pm}\right) \approx 100 \%(\ell=e, \mu)$. Using the like-sign dilepton (LSD) final state, the current lower bound on $m_{H^{ \pm \pm}}$varies between 770 and $870 \mathrm{GeV}$ [86] at the $95 \%$ CL from the direct search for a doubly-charged Higgs boson in the $13 \mathrm{TeV}$ LHC run. The corresponding lower limit on $m_{H^{ \pm \pm}}$changes significantly for $v_{T} \geq 10^{-4} \mathrm{GeV}$ ( small $Y_{v}$ ), when the LSD decay mode of $H^{ \pm \pm}$is highly suppressed while several competing decay modes of $H^{ \pm \pm}$start opening up, such as (i) a pair of heavy bosons $W^{ \pm} W^{ \pm}$, (ii) $W^{ \pm} H^{ \pm}$ and (iii) $H^{ \pm} H^{ \pm}$, if these are kinematically accessible. The subsequent decays of $W$ and $H^{ \pm}$into various leptons and jets give rise to rather complicated final states. Due to the cascade nature of the final state, the collider bound on the doubly-charged scalar is rather weak in this case.

The ATLAS collaboration studied the pair production of doubly-charged scalars that subsequently decay into pairs of $W$ bosons (assuming $\operatorname{Br}\left(H^{ \pm \pm} \rightarrow W^{ \pm} W^{ \pm}\right) \approx 100 \%$ ) in the $13 \mathrm{TeV}$ LHC run. Non-observation of any signal beyond the standard model background sets a new limit on the doublycharged scalar mass. Using a data sample from an integrated luminosity of $36.1 \mathrm{fb}^{-1}$, the $m_{H^{ \pm \pm}}$has been excluded between 200 and $220 \mathrm{GeV}$ at 95\% CL [87].

The left-right (LR) symmetric model predicts two types of doubly-charged scalars $H_{L}^{ \pm \pm}$and $H_{R}^{ \pm \pm}$, corresponding to $S U(2)_{L}$ and $S U(2)_{R}$ triplet scalars $\Delta_{L}$ and $\Delta_{R}$, respectively. As these two scalars belong to different gauge group, their couplings with fermions and gauge bosons are distinctly different. This is reflected in their production rates at the LHC: $\sigma\left(p p \rightarrow H_{L}^{++} H_{L}^{--}+\cdots\right) \approx 2.3 \times \sigma(p p \rightarrow$ $\left.H_{R}^{++} H_{R}^{--}\right)+\cdots$, due to the different coupling strength of $H_{L, R}^{ \pm \pm}$with the $Z$ boson [86,88]. The ATLAS Collaboration looked for doubly-charged scalars in the LSD invariant mass distributions for the $e^{ \pm} e^{ \pm}, \mu^{ \pm} \mu^{ \pm}$and $e^{ \pm} \mu^{ \pm}$final states, and also in final states with three or four leptons (only electrons and muons) in the $13 \mathrm{TeV}$ LHC run with an integrated luminosity of $36.1 \mathrm{fb}^{-1}$. No significant excess over the standard model prediction was observed. As a result, lower mass limits were obtained for the $m_{H_{L}^{ \pm \pm}}$and $m_{H_{R}^{ \pm \pm}}$, assuming $\operatorname{Br}\left(H_{L, R}^{ \pm \pm} \rightarrow \ell^{ \pm} \ell^{ \pm}\right)=100 \%(\ell=e, \mu)$. The limit for $m_{H_{L}^{ \pm \pm}}$is same as that in the Type-II seesaw model. However, for the $m_{H_{R}^{ \pm \pm}}$the observed lower limit varies between 660 and $760 \mathrm{GeV}$ at the $95 \% \mathrm{CL}[86]$.

In the GM model, the current LHC limit on $m_{H^{ \pm \pm}}$varies between 200 and $220 \mathrm{GeV}$ at $95 \% \mathrm{CL}$, as obtained using an integrated luminosity of $36.1 \mathrm{fb}^{-1}$ in the $13 \mathrm{TeV}$ LHC run by the ATLAS Collaboration [87].

One should note that in all these search analyses only prompt decays of $H_{L, R}^{ \pm \pm}$scalars $(c \tau<10 \mu \mathrm{m})$ were considered [86]. Hence they are complementary to the long-lived particle search that is possible with MoEDAL. In the Type-II seesaw model, there are certain regions of the $\left(v_{T}, m_{H^{ \pm \pm}}\right)$ parameter plane where the life-time of the doubly-charged scalar can be much longer. If the $H^{ \pm \pm}$were to decay outside an LHC detector, it would leave a heavily-ionizing charged track signal. Both the ATLAS and CMS collaborations have studied these signatures of such heavy long-lived particles, as we discuss later.

\subsection{Run 3 projections for doubly-charged particles}

We study here the prospective MoEDAL sensitivities to four types of doubly-charged particles: (singlet, triplet) $\times$ (scalar, fermion). For the singlet and triplet types, the weak gauge quantum number assignments are $\left(S U(2)_{L}, U(1)_{Y}\right)=$ $(\mathbf{1}, 2)$ and $(\mathbf{3}, 1)$, respectively, and all these particles are assumed to be colour singlets ${ }^{10}$.

\footnotetext{
10 We do not consider heavy coloured particles that hadronize into doubly-charged particles. These would have large production crosssections due to their colour charge and their production velocity could be small, facilitating discovery by MoEDAL.
} 

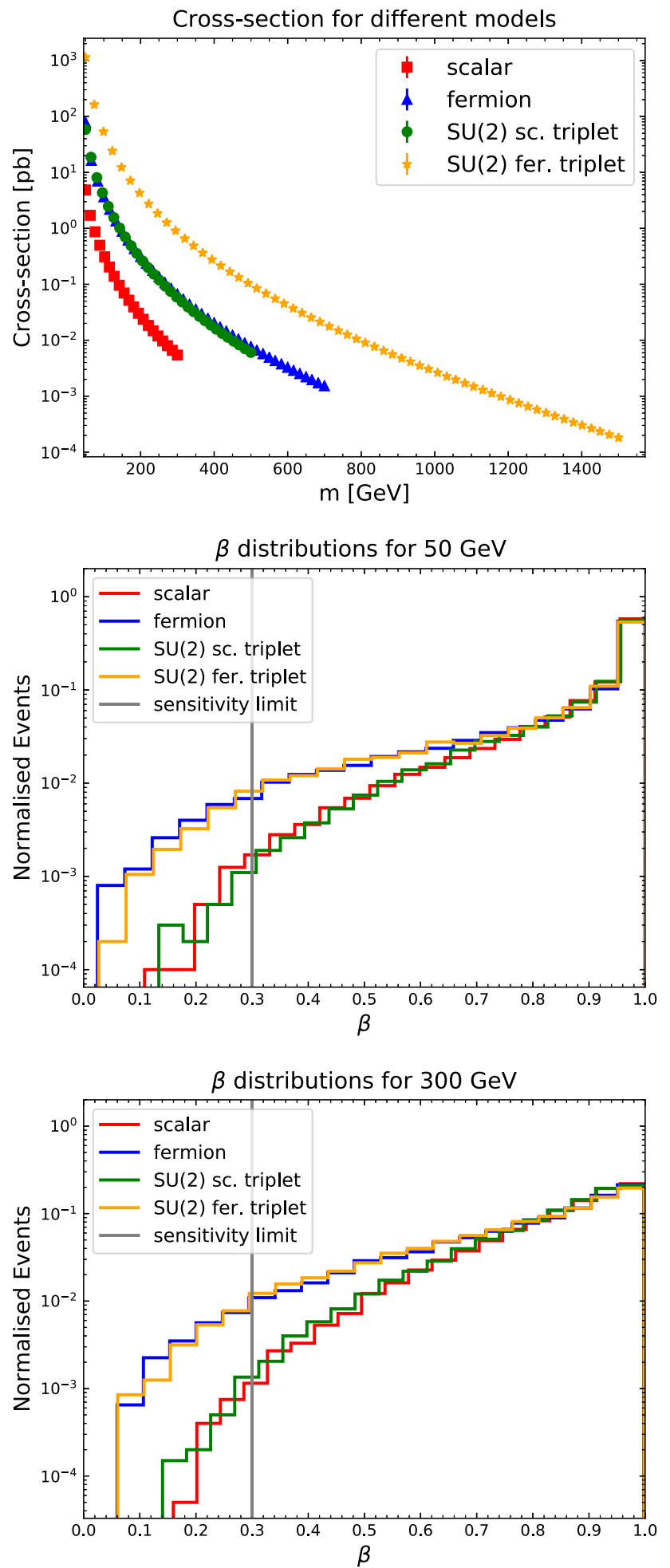

Fig. 4 Top panel: the leading-order production cross-sections at 13 $\mathrm{TeV}$ for various types of doubly-charged particles. Middle and lower panels: the velocity distributions for various doubly-charged particles with masses 50 and $300 \mathrm{GeV}$

We show in the top panel of Fig. 4 the $13 \mathrm{TeV}$ crosssections for the production of doubly-charged particles. We see that, for species with the same spin, the cross-sections for triplet particles are an order of magnitude larger than those for the singlet. This is because we included all the neutral $p p \rightarrow Y^{0} Y^{0}, Y^{+} Y^{-}, Y^{++} Y^{--}+\cdots$ and charged $p p \rightarrow Y^{0} Y^{ \pm}, Y^{ \pm} Y^{ \pm \pm}+\cdots$ triplet production modes, assuming that the heavier components of the multiplets decay promptly into the nearly-degenerate lightest doubly-charged partner, so that all the production modes end up with the final state containing two doubly-charged particles. The neutral charge combinations are produced via $s$-channel $\gamma / Z$, while the charged ones are produced via an $s$-channel $W^{ \pm}$. Unlike the triplet species, the singlet species has only one neutral production mode, namely $p p \rightarrow Y^{++} Y^{--}+\cdots$.

We also see in the top panel of Fig. 4 that the cross-sections are an order of magnitude higher for fermions than scalars with the same gauge quantum numbers. One reason for this is that a Dirac fermion has twice as many degrees of freedom as a complex scalar. Another reason is, as already discussed in Sect. 5.2, the production of doubly-charged particles is mediated by $s$-channel gauge boson exchange, in which the two-scalar final states suffer threshold velocity suppression: $\sigma \rightarrow 0$ in the limit $\beta \rightarrow 0$. This suppression is absent for fermion pair production.

In the case of doubly-charged particles, the threshold velocity for detection is $\beta_{\mathrm{th}}=0.3$, higher by a factor of two compared to singly-charged ones due to the higher electric charge. In the middle and bottom panels of Fig. 4 we show the normalised velocity distributions for pair-produced doubly-charged particles with masses $m_{Y}=50$ and $300 \mathrm{GeV}$, respectively. The grey vertical lines indicate $\beta_{\text {th }}=0.3$, and only events to the left of the lines are detectable by MoEDAL. Comparing these two plots, we see that the distributions are more mass-dependent in the high- $\beta$ region, whereas the mass effects are mild in the lower-velocity regions where $\beta \lesssim 0.3$. Nevertheless, about a factor of two more events satisfy $\beta<0.3$ for $m_{Y}=300 \mathrm{GeV}$ than for $m_{Y}=50 \mathrm{GeV}$. We also see clear differences in the momentum distributions between fermions and scalar particles for both $m_{Y}=50$ and $300 \mathrm{GeV}$. As discussed earlier, this is because angularmomentum conservation again forces the scalar pair production via the $s$-channel gauge boson exchange to be velocity suppressed, $\sigma \rightarrow 0$ in the limit $\beta \rightarrow 0$. Unlike scalars, the production rate for fermions is non-vanishing even at $\beta=0$.

We show in Fig. 5 the expected MoEDAL sensitivities for four types of colour-singlet doubly-charged particles: a scalar singlet (red), a scalar triplet (blue), a fermion singlet (green) and a fermion triplet (magenta), assuming a Run 3 integrated luminosity of $30 \mathrm{fb}^{-1}$. As expected from the above discussion, the fermion triplet has the highest mass reach among the four types, due to its large cross-section and favourable production velocities. MoEDAL can probe this particle up to $m_{Y} \sim 1130$ (990) $\mathrm{GeV}$ with $N_{\text {sig }}=1$ (2) for the Run 3 luminosity if $c \tau \gtrsim 100 \mathrm{~m}$, as indicated by the 


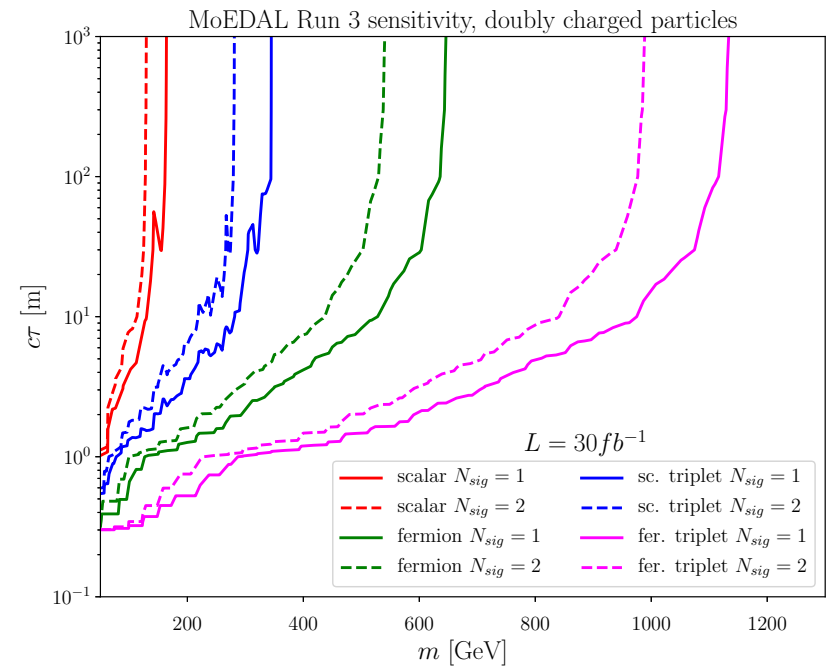

Fig. 5 The expected sensitivities of MoEDAL for various long-lived doubly-charged particle species, assuming $30 \mathrm{fb}^{-1}$ of integrated luminosity

solid (dashed) line. The next most sensitive particle is the fermion-singlet, for which we estimate the MoEDAL mass reach with $c \tau \gtrsim 100 \mathrm{~m}$ to be around 650 (540) GeV for $N_{\text {sig }}=1$ (2). The mass reaches for the two scalar particles (singlet and triplet) are significantly lower than the corresponding fermionic particles, due to their smaller crosssections and higher typical velocities. The MoEDAL mass reaches at Run 3 are around 340 (280) GeV for the scalar triplet and 160 (130) GeV for the scalar singlet for $N_{\text {sig }}=1$ (2) if $c \tau \gtrsim 100 \mathrm{~m}$.

\subsection{Comparison with the existing searches}

We compare prospective MoEDAL mass reaches $\left(N_{\text {sig }}=1\right)$ at Run 3 for the types of doubly-charged meta-stable particles examined in this section, together with the available mass bound. As discussed in Sect. 5.3, the most relevant constraints are obtained by the heavy stable charged particle (HSCP) searches by ATLAS and CMS, which are based on the ionization energy loss $d E / d x$ and the time-of-flight measurements but incorporate additional trigger requirements. The analysis utilizing the largest data-set $\left(L=36.1 \mathrm{fb}^{-1}\right)$ is that from ATLAS [54]. In Sect. 5.3 we compared the MoEDAL mass reaches for various long-lived sparticles with mass bounds from this analysis. However, ATLAS did not interpret their results for doubly-charged particles in [54], and did not provide mass limits.

CMS, on the other hand, has published their latest HSCP analysis [55] based on a smaller data-set, $L=2.5 \mathrm{fb}^{-1}$, and have interpreted their result for a long-lived doubly-charged particle that is an $\mathrm{SU}(2)$ singlet and has spin-1/2. The mass bound for this particle is found to be $630 \mathrm{GeV}$, which is 20 $\mathrm{GeV}$ lower than the MoEDAL Run 3 mass reach, as shown in
Table 2 Compilation of the prospective MoEDAL mass reaches $\left(N_{\text {sig }}=1\right)$ at Run 3 and comparison with the current $(95 \% \mathrm{CL})$ mass bound for a long-lived fermion-singlet doubly-charged particle obtained from the CMS heavy stable charged particle search with $L=2.5 \mathrm{fb}^{-1}$ [55] (in parentheses). Our estimates of possible CMS bounds on other types of particle are in double parentheses. All masses are in GeV

\begin{tabular}{lcl}
\hline & MoEDAL & $(\mathrm{CMS})$ \\
\hline Scalar singlet & 160 & $((320))$ \\
Fermion singlet & 650 & $(630)$ \\
Scalar triplet & 340 & $((590))$ \\
Fermion triplet & 1130 & $((900))$ \\
\hline
\end{tabular}

parentheses in Table 2. We emphasis that the CMS analysis incorporated extra assumptions, such as an $E_{\mathrm{T}}^{\mathrm{miss}}>170 \mathrm{GeV}$ requirement or the presence of a muon-like particle, rendering the result more model-dependent than the prospective MoEDAL sensitivity.

In [55], CMS did not directly interpret their result for other types of doubly-charged particles. In order to derive approximate mass bounds for other types of doubly-charged particles, we assume that the detection efficiencies are not very sensitive to the nature of particles other than the electric charge. With this assumption we use the cross-section upper limit for the fermion singlet particle provided in [55] as approximate cross-section limits for other types of doublycharged particles (scalar singlet, scalar triplet and fermion triplet).

We estimate the CMS mass bound for scalar singlet (scalar triplet) obtained by the above recasting procedure to be $\sim 320$ (590) GeV, which can be compared with the corresponding MoEDAL mass reach of $160(340) \mathrm{GeV}\left(N_{\mathrm{sig}}=1\right.$, $L=30 \mathrm{fb}^{-1}$ ). In the case of the fermion triplet, we estimate the CMS mass bound to be $\sim 900 \mathrm{GeV}$, which is surpassed by the Run 3 mass reach of MoEDAL, which is $1130 \mathrm{GeV}$. Our estimates of these CMS sensitivities are shown in double parentheses, in view of potential model dependence associated with the CMS analysis assumptions and the fact that they are not official CMS results and have uncertainties due to our recasting procedure.

\section{Conclusions}

We have analysed in this paper the prospective sensitivities of the MoEDAL detector for searches for singly- and doublycharged long-lived particles during Run 3 of the LHC, considering the specific examples of supersymmetric particles and scalars and fermions that are suggested by Type-II and Type-III seesaw models of neutrino masses, respectively. We emphasise that the MoEDAL search would be completely model-independent, using simply the capabilities of 
its NTDs. However, MoEDAL suffers from two disadvantages with respect to the general-purpose LHC detectors, ATLAS and CMS. It is sensitive only to an anomalous level of ionisation corresponding to velocities $\beta<0.15$ for singlycharged particles and $\beta<0.3$ for doubly-charged particles, and it is anticipated that MoEDAL may accumulate $30 \mathrm{fb}^{-1}$ of luminosity by the end of Run 3, an order of magnitude less than ATLAS and CMS.

The greatest MoEDAL sensitivities for supersymmetric particles are for strongly-interacting species, namely lightflavoured squarks, the gluino and stop squarks. There are also interesting sensitivities for long-lived charginos, whereas the sensitivity for a long-lived slepton is below the modelindependent limit already established by LEP. However, we regard the model-independent potential MoEDAL sensitivities to other sparticles as quite complementary to the limits set by ATLAS and CMS, and of particular interest in scenarios such as those with weakly-broken R-parity in which there is no $E_{\mathrm{T}}^{\mathrm{miss}}$.

Among the particles examined in this paper, the doublycharged fermion triplet appears to be the most favourable for MoEDAL. Since it is doubly-charged, the threshold velocity is relaxed to $\beta=0.3$, so MoEDAL accepts a larger fraction of signal events. Since it is a fermion, the particles are much more likely to be produced near threshold than would be a scalar. Finally, it is an SU(2)-triplet and has the largest cross section among the doubly-charged particles we have studied. Therefore, the typical mass scale that can be probed is higher than for the other electroweakly-interacting cases, corresponding to a lower typical production velocity. Although our study suggests that the MoEDAL can probe a substantial non-excluded region of the parameter space of the fermiontriplet doubly-charged particle, one must keep in mind that the estimated CMS limit is approximate and based on a much smaller data set than that obtained by the end of Run 2 .

It is clear that by the end of Run 3 ATLAS and CMS are likely to be able to provide better constraints on all the longlived charged particle candidates that we have considered here. However, we would like to emphasise that the MoEDAL analysis we have described here would be able to set limits that are independent of auxiliary signal assumptions.

Acknowledgements The work of J.E. was supported by the UK STFC Grant ST/P000258/1 and by the Estonian Research Council via a Mobilitas Pluss grant. D.K.G. would like to thank the High Energy, Cosmology and Astroparticle Physics Division of ICTP, Trieste for hospitality during while part of this work was started. The work of R.M. is partially supported by the National Science Centre, Poland, under research Grants 2017/26/E/ST2/00135. The work of K.S. is partially supported by the National Science Centre, Poland, under research Grants 2017/26/E/ST2/00135 and the Beethoven grants DEC2016/23/G/ST2/04301. D.K.G would like to thank N. Ghosh and I. Saha for discussions.
Data Availability Statement This manuscript has no associated data or the data will not be deposited. [Authors' comment: All results are presented in the figures in the manuscript.]

Open Access This article is licensed under a Creative Commons Attribution 4.0 International License, which permits use, sharing, adaptation, distribution and reproduction in any medium or format, as long as you give appropriate credit to the original author(s) and the source, provide a link to the Creative Commons licence, and indicate if changes were made. The images or other third party material in this article are included in the article's Creative Commons licence, unless indicated otherwise in a credit line to the material. If material is not included in the article's Creative Commons licence and your intended use is not permitted by statutory regulation or exceeds the permitted use, you will need to obtain permission directly from the copyright holder. To view a copy of this licence, visit http://creativecomm ons.org/licenses/by/4.0/.

Funded by $\mathrm{SCOAP}^{3}$.

\section{References}

1. L. Lee, C. Ohm, A. Soffer, T. Yu, Collider searches for long-lived particles beyond the Standard Model. Prog. Part. Nucl. Phys. 106, 210-255 (2019). arXiv:1810.12602 [hep-ph]

2. J. Alimena at al., Searching for long-lived particles beyond the Standard Model at the large hadron collider. arXiv:1903.04497 [hep-ex]

3. D. Curtin et al., Long-lived particles at the energy frontier: the MATHUSLA physics case. Rep. Prog. Phys. 82(11), 116201 (2019). arXiv:1806.07396 [hep-ph]

4. B. Acharya et al. [MoEDAL Collaboration], Search for magnetic monopoles with the MoEDAL prototype trapping detector in 8 $\mathrm{TeV}$ proton-proton collisions at the LHC. JHEP 08, 067 (2016). arXiv:1604.06645 [hep-ex]

5. B. Acharya et al., Search for magnetic monopoles with the MoEDAL forward trapping detector in $13 \mathrm{TeV}$ proton-proton collisions at the LHC. Phys. Rev. Lett. 118(6), 061801 (2017). arXiv: 1611.06817 [hep-ex]

6. B. Acharya et al., Search for magnetic monopoles with the MoEDAL forward trapping detector in $2.11 \mathrm{fb}^{-1}$ of $13 \mathrm{TeV}$ protonproton collisions at the LHC. Phys. Lett. B 782, 510-516 (2018). arXiv:1712.09849 [hep-ex]

7. B. Acharya et al., Magnetic monopole search with the full MoEDAL trapping detector in $13 \mathrm{TeV}$ pp collisions interpreted in photon-fusion and Drell-Yan production. Phys. Rev. Lett. 123(2), 021802 (2019). arXiv: 1903.08491 [hep-ex]

8. B. Acharya et al. [MoEDAL Collaboration], First search for dyons with the full MoEDAL trapping detector in $13 \mathrm{TeV}$ pp collisions. arXiv:2002.00861 [hep-ex]

9. G. Aad et al. [ATLAS Collaboration], Search for magnetic monopoles in $\sqrt{s}=7 \mathrm{TeV} p p$ collisions with the ATLAS detector. Phys. Rev. Lett. 109, 261803 (2012). arXiv:1207.6411 [hep-ex]

10. G. Aad et al., Search for magnetic monopoles and stable particles with high electric charges in $8 \mathrm{TeV} p p$ collisions with the ATLAS detector. Phys. Rev. D 93(5), 052009 (2016). arXiv:1509.08059 [hep-ex]

11. B. Acharya et al. [MoEDAL], The physics programme of the MoEDAL experiment at the LHC. Int. J. Mod. Phys. A 29, 1430050 (2014). arXiv:1405.7662 [hep-ph]

12. D. Felea, J. Mamuzic, R. Masełek, N. Mavromatos, V. Mitsou, J. Pinfold, R. Ruiz de Austri, K. Sakurai, A. Santra, O. Vives, Prospects for discovering supersymmetric long-lived particles with MoEDAL. arXiv:2001.05980 [hep-ph] 
13. K. Sakurai, D. Felea, J. Mamuzic, N. Mavromatos, V. Mitsou, J. Pinfold, R. Ruiz de Austri, A. Santra, O. Vives, SUSY discovery prospects with MoEDAL. arXiv:1903.11022 [hep-ph]

14. ATLAS Collaboration, https://twiki.cern.ch/twiki/bin/view/ AtlasPublic/SupersymmetryPublicResults

15. CMS Collaboration, https://twiki.cern.ch/twiki/bin/view/ CMSPublic/PhysicsResultsSUS

16. S.P. Martin, A Supersymmetry primer. Adv. Ser. Direct. High Energy Phys. 21, 1 (2010). arXiv:hep-ph/9709356. [Adv. Ser. Direct. High Energy Phys. 18, 1 (1998)]

17. H.E. Haber, G.L. Kane, The search for supersymmetry: probing physics beyond the Standard Model. Phys. Rep. 117, 75 (1985)

18. M. Drees, R. Godbole, P. Roy, Theory and Phenomenology of Sparticles: An Account of Four-dimensional N=1 Supersymmetry in High Energy Physics (World Scientific, Hackensack, 2004), p. 555

19. H. Baer, X. Tata, Weak Scale Supersymmetry: From superfields to Scattering Events (Cambridge University Press, Cambridge, 2006), p. 556

20. R. Barbieri et al., R-parity violating supersymmetry. Phys. Rep. 420, 1 (2005). arXiv:hep-ph/0406039

21. K. Barry, P.W. Graham, S. Rajendran, Displaced vertices from $R$-parity violation and baryogenesis. Phys. Rev. D 89(5), 054003 (2014). arXiv:1310.3853 [hep-ph]

22. N. Arkani-Hamed, S. Dimopoulos, Supersymmetric unification without low energy supersymmetry and signatures for fine-tuning at the LHC. JHEP 0506, 073 (2005). arXiv:hep-th/0405159

23. G.F. Giudice, A. Romanino, Split supersymmetry. Nucl. Phys. B 699, 65 (2004). Erratum: [Nucl. Phys. B 706, 487 (2005)]. arXiv:hep-ph/0406088

24. L. Randall, R. Sundrum, Out of this world supersymmetry breaking. Nucl. Phys. B 557, 79 (1999). arXiv:hep-th/9810155

25. G.F. Giudice, M.A. Luty, H. Murayama, R. Rattazzi, Gaugino mass without singlets. JHEP 9812, 027 (1998). arXiv:hep-ph/9810442

26. G.F. Giudice, R. Rattazzi, Theories with gauge mediated supersymmetry breaking. Phys. Rep. 322, 419 (1999). arXiv:hep-ph/9801271

27. G.R. Farrar, P. Fayet, Phenomenology of the production, decay, and detection of new hadronic states associated with supersymmetry. Phys. Lett. B 76, 575-579 (1978)

28. R. Foot, H. Lew, X. He, G.C. Joshi, Seesaw neutrino masses induced by a triplet of leptons. Z. Phys. C 44, 441 (1989)

29. B. Bajc, G. Senjanovic, Seesaw at LHC. JHEP 08, 014 (2007). arXiv:hep-ph/0612029

30. A. Arhrib, B. Bajc, D.K. Ghosh, T. Han, G. Huang, I. Puljak, G. Senjanovic, Collider signatures for heavy lepton triplet in Type I+III seesaw. Phys. Rev. D 82, 053004 (2010). arXiv:0904.2390 [hep-ph]

31. M. Cirelli, N. Fornengo, A. Strumia, Minimal dark matter. Nucl. Phys. B 753, 178-194 (2006). arXiv:hep-ph/0512090

32. S. Jana, N. Okada, D. Raut, Displaced vertex and disappearing track signatures in type-III seesaw. arXiv:1911.09037 [hep-ph]

33. J. Schechter, J.W.F. Valle, Neutrino masses in SU(2) x U(1) theories. Phys. Rev. D 22, 2227 (1980)

34. M. Magg, C. Wetterich, Neutrino mass problem and gauge hierarchy. Phys. Lett. 94B, 61 (1980)

35. T.P. Cheng, L.F. Li, Neutrino masses, mixings and oscillations in $\mathrm{SU}(2) \times \mathrm{U}(1)$ models of electroweak interactions. Phys. Rev. D 22, 2860 (1980)

36. G. Lazarides, Q. Shafi, C. Wetterich, Proton lifetime and fermion masses in an SO(10) model. Nucl. Phys. B 181, 287 (1981)

37. R.N. Mohapatra, G. Senjanovic, Neutrino masses and mixings in gauge models with spontaneous parity violation. Phys. Rev. D 23, 165 (1981)

38. M. Lindner, M. Platscher, F.S. Queiroz, A call for new physics: the muon anomalous magnetic moment and lepton flavor violation. Phys. Rep. 731, 1 (2018). arXiv:1610.06587 [hep-ph]
39. A. Melfo, M. Nemevsek, F. Nesti, G. Senjanovic, Y. Zhang, Type II seesaw at LHC: the roadmap. Phys. Rev. D 85, 055018 (2012). arXiv:1108.4416 [hep-ph]

40. P. Bhupal Dev, D.K. Ghosh, N. Okada, I. Saha, JHEP 03, 150 (2013). https://doi.org/10.1007/JHEP03(2013)150. arXiv:1301.3453 [hep-ph]

41. D.K. Ghosh, N. Ghosh, I. Saha, A. Shaw, Phys. Rev. D 97(11), 115022 (2018). https://doi.org/10.1103/PhysRevD.97. 115022. arXiv:1711.06062 [hep-ph]

42. R. Kuchimanchi, R. Mohapatra, No parity violation without R-parity violation. Phys. Rev. D 48, 4352-4360 (1993). arXiv:hep-ph/9306290

43. K. Babu, R.N. Mohapatra, Minimal supersymmetric left-right model. Phys. Lett. B 668, 404-409 (2008). arXiv:0807.0481 [hep$\mathrm{ph}]$

44. R. Francis, M. Frank, C.S. Kalman, Anomalous magnetic moment of the muon arising from the extensions of the supersymmetric standard model based on left-right symmetry. Phys. Rev. D 43, 2369-2385 (1991)

45. K. Huitu, J. Maalampi, M. Raidal, Slepton pair production in $e^{+} e^{-}$ collision in supersymmetric left-right model. Phys. Lett. B 328, 60-66 (1994). arXiv:hep-ph/9402219

46. M. Frank, D.K. Ghosh, K. Huitu, S.K. Rai, I. Saha, H. Waltari, Left-right supersymmetry after the Higgs boson discovery. Phys. Rev. D 90(11), 115021 (2014). arXiv:1408.2423 [hep-ph]

47. A. Delgado, C. Garcia Cely, T. Han, Z. Wang, Phenomenology of a lepton triplet. Phys. Rev. D 84, 073007 (2011). (and references therein)

48. A. Alloul, M. Frank, B. Fuks, M. Rausch de Traubenberg, Doublycharged particles at the large hadron collider. Phys. Rev. D 88, 075004 (2013). (and references therein)

49. P. Bhupal Dev, Y. Zhang, Displaced vertex signatures of doubly charged scalars in the type-II seesaw and its left-right extensions. JHEP 10, 199 (2018). arXiv:1808.00943 [hep-ph]

50. S. Antusch, O. Fischer, A. Hammad, C. Scherb, Low scale type II seesaw: present constraints and prospects for displaced vertex searches. JHEP 02, 157 (2019). arXiv:1811.03476 [hep-ph]

51. S. Banerjee, B. Bhattacherjee, A. Goudelis, B. Herrmann, D. Sengupta, R. Sengupta, Determining the lifetime of long-lived particles at the LHC. arXiv:1912.06669 [hep-ph]

52. O. Bulekov, M.Y. Khlopov, A. Romaniouk, Y.S. Smirnov, Search for double charged particles as direct test for dark atom constituents. Bled Workshops Phys. 18(2), 11-24 (2017). arXiv:1711.10773 [hep-ph]

53. M. Aaboud et al. [ATLAS Collaboration], Search for heavy charged long-lived particles in proton-proton collisions at $\sqrt{s}=13 \mathrm{TeV}$ using an ionisation measurement with the ATLAS detector. Phys. Lett. B 788, 96 (2019). arXiv:1808.04095 [hep-ex]

54. M. Aaboud et al. [ATLAS Collaboration], Search for heavy charged long-lived particles in the ATLAS detector in $36.1 \mathrm{fb}^{-1}$ of protonproton collision data at $\sqrt{s}=13 \mathrm{TeV}$. Phys. Rev. D 99(9), 092007 (2019). arXiv:1902.01636 [hep-ex]

55. V. Khachatryan et al. [CMS Collaboration], Search for long-lived charged particles in proton-proton collisions at $\sqrt{s}=13 \mathrm{TeV}$. Phys. Rev. D 94(11), 112004 (2016). arXiv:1609.08382 [hep-ex]

56. M. Aaboud et al. [ATLAS Collaboration], Search for heavy longlived multicharged particles in proton-proton collisions at $\sqrt{s}=13$ TeV using the ATLAS detector. Phys. Rev. D 99(5), 052003 (2019). arXiv:1812.03673 [hep-ex]

57. S. Chatrchyan et al. [CMS Collaboration], Searches for long-lived charged particles in $p p$ collisions at $\sqrt{s}=7$ and 8 TeV. JHEP 1307, 122 (2013). arXiv:1305.0491 [hep-ex]

58. C. Biggio, E. Fernandez-Martinez, M. Filaci, J. HernandezGarcia, J. Lopez-Pavon, Global bounds on the type-III seesaw. arXiv:1911.11790 [hep-ph] 
59. J. Kang, M.A. Luty, Macroscopic strings and 'quirks' at colliders. JHEP 0911, 065 (2009). https://doi.org/10.1088/1126-6708/2009/ 11/065. arXiv:0805.4642 [hep-ph]

60. M. Aaboud et al. [ATLAS Collaboration], Search for longlived, massive particles in events with displaced vertices and missing transverse momentum in $\sqrt{s}=13 \mathrm{TeV} p p$ collisions with the ATLAS detector. Phys. Rev. D 97(5), 052012 (2018). arXiv:1710.04901 [hep-ex]

61. M. Aaboud et al. [ATLAS Collaboration], Search for long-lived charginos based on a disappearing-track signature in pp collisions at $\sqrt{s}=13 \mathrm{TeV}$ with the ATLAS detector. JHEP 1806, 022 (2018). arXiv: 1712.02118 [hep-ex]

62. A.M. Sirunyan et al. [CMS Collaboration], Search for long-lived particles with displaced vertices in multijet events in proton-proton collisions at $\sqrt{s}=13$ TeV. Phys. Rev. D 98(9), 092011 (2018). arXiv:1808.03078 [hep-ex]

63. A.M. Sirunyan et al., [CMS Collaboration], Search for disappearing tracks in proton-proton collisions at $\sqrt{s}=13 \mathrm{TeV}$. arXiv:2004.05153 [hep-ex]

64. A.M. Sirunyan et al. [CMS Collaboration], Search for evidence of the type-III seesaw mechanism in multilepton final states in protonproton collisions at $\sqrt{s}=13 \mathrm{TeV}$. Phys. Rev. Lett. 119(22), 221802 (2017). arXiv:1708.07962 [hep-ex]

65. ATLAS Collaboration, Generation and Simulation of R-Hadrons in the ATLAS Experiment. ATL-PHYS-PUB-2019-019

66. J. Alwall et al., The automated computation of tree-level and next-to-leading order differential cross sections, and their matching to parton shower simulations. JHEP 1407, 079 (2014). arXiv:1405.0301 [hep-ph]

67. N.D. Christensen, C. Duhr, FeynRules-Feynman rules made easy. Comput. Phys. Commun. 180, 1614 (2009). arXiv:0806.4194 [hep$\mathrm{ph}]$

68. T. Jittoh, J. Sato, T. Shimomura, M. Yamanaka, Long life stau in the minimal supersymmetric standard model. Phys. Rev. D 73, 055009 (2006). https://doi.org/10.1103/PhysRevD.73.055009. arXiv:hep-ph/0512197. Erratum: [Phys. Rev. D 87(1), 019901 (2013)]

69. S. Kaneko, J. Sato, T. Shimomura, O. Vives, M. Yamanaka, Measuring lepton flavor violation at LHC with a long-lived slepton in the coannihilation region. Phys. Rev. D 78(11), 116013 (2008). https:// doi.org/10.1103/PhysRevD.78.116013. arXiv:0811.0703 [hep-ph] Erratum: [Phys. Rev. D 87(3), 039904 (2013)]

70. Y. Konishi, S. Ohta, J. Sato, T. Shimomura, K. Sugai, M. Yamanaka, First evidence of the constrained minimal supersymmetric standard model is appearing soon. Phys. Rev. D 89(7), 075006 (2014). https://doi.org/10.1103/PhysRevD.89. 075006. arXiv:1309.2067 [hep-ph]

71. LHC SUSY Cross Section Working Group, https://twiki.cern.ch/ twiki/bin/view/LHCPhysics/SUSYCrossSections

72. W. Beenakker, C. Borschensky, M. Krämer, A. Kulesza, E. Laenen, NNLL-fast: predictions for coloured supersymmetric particle production at the LHC with threshold and Coulomb resummation. JHEP 1612, 133 (2016). arXiv:1607.07741 [hep-ph]
73. B. Fuks, M. Klasen, D.R. Lamprea, M. Rothering, Precision predictions for electroweak superpartner production at hadron colliders with Resummino. Eur. Phys. J. C 73, 2480 (2013). arXiv:1304.0790 [hep-ph]

74. LEP2 SUSY Working Group, Stable heavy charged particles. http://lepsusy.web.cern.ch/lepsusy/www/stable_summer02/ stable_208.html

75. J.C. Pati, A. Salam, Lepton number as the fourth color. Phys. Rev. D 10, 275 (1974). Erratum: [Phys. Rev. D 11, 703 (1975)]

76. R.N. Mohapatra, J.C. Pati, Left-right gauge symmetry and an isoconjugate model of CP violation. Phys. Rev. D 11, 566 (1975)

77. G. Senjanovic, R.N. Mohapatra, Exact left-right symmetry and spontaneous violation of parity. Phys. Rev. D 12, 1502 (1975)

78. H. Georgi, M. Machacek, Doubly charged Higgs bosons. Nucl. Phys. B 262, 463 (1985)

79. M.S. Chanowitz, M. Golden, Higgs boson triplets with $\mathbf{M}(W)=$ $\mathrm{M}(Z) \cos \theta_{W}$. Phys. Lett. 165B, 105 (1985)

80. J.F. Gunion, R. Vega, J. Wudka, Higgs triplets in the standard model. Phys. Rev. D 42, 1673 (1990)

81. J.F. Gunion, R. Vega, J. Wudka, Naturalness problems for rho $=1$ and other large one loop effects for a standard model Higgs sector containing triplet fields. Phys. Rev. D 43, 2322 (1991)

82. A. Ismail, H.E. Logan, Y. Wu, Updated constraints on the GeorgiMachacek model from LHC Run 2. arXiv:2003.02272 [hep-ph] (and references therein)

83. J.E. Cieza Montalvo, N.V. Cortez, J. Sa Borges, M.D. Tonasse, Searching for doubly charged Higgs bosons at the LHC in a 3-3-1 model. Nucl. Phys. B 756, 1 (2006). Erratum: [Nucl. Phys. B 796, $422(2008)]$

84. A. Alves, E. Ramirez Barreto, A.G. Dias, C.A. de S.Pires, F.S. Queiroz, P.S. Rodrigues da Silva, Probing 3-3-1 models in diphoton Higgs boson decay. Phys. Rev. D 84, 115004 (2011)

85. N. Arkani-Hamed, A.G. Cohen, E. Katz, A.E. Nelson, T. Gregoire, J.G. Wacker, The minimal moose for a little Higgs. JHEP 0208, 021 (2002)

86. M. Aaboud et al. [ATLAS Collaboration], Search for doubly charged Higgs boson production in multi-lepton final states with the ATLAS detector using proton-proton collisions at $\sqrt{s}=13 \mathrm{TeV}$. Eur. Phys. J. C 78(3), 199 (2018). arXiv:1710.09748 [hep-ex]

87. M. Aaboud et al. [ATLAS Collaboration], Search for doubly charged scalar bosons decaying into same-sign $W$ boson pairs with the ATLAS detector. Eur. Phys. J. C 79(1), 58 (2019). arXiv: 1808.01899 [hep-ex]

88. K. Huitu, J. Maalampi, A. Pietila, M. Raidal, Doubly charged Higgs at LHC. Nucl. Phys. B 487, 27 (1997) 